\title{
Threshold Defined Camouflaged Gates in 65nm Technology for Reverse Engineering Protection
}

\author{
Anirudh S Iyengar \\ School of Electrical Engineering and \\ Computer Science \\ The Pennsylvania State University \\ University Park, Pennsylvania \\ asi7@psu.edu \\ Swaroop Ghosh \\ School of Electrical Engineering and \\ Computer Science \\ The Pennsylvania State University \\ University Park, Pennsylvania \\ szg212@psu.edu
}

\author{
Deepak Vontela \\ Computer Science and Engineering \\ University of South Florida \\ Tampa, Florida \\ deepakreddy@mail.usf.edu
}

Syedhamidreza Motaman

School of Electrical Engineering and Computer Science

The Pennsylvania State University

University Park, Pennsylvania

sxm88@ psu.edu

\author{
Ithihasa Reddy \\ Computer Science and Engineering \\ University of South Florida \\ Tampa, Florida \\ ithihasaredd@mail.usf.edu
}

\author{
Jae-won Jang \\ School of Electrical Engineering and \\ Computer Science \\ The Pennsylvania State University \\ University Park, Pennsylvania \\ jxj328@ psu.edu
}

\begin{abstract}
Due to the ever-increasing threat of Reverse Engineering (RE) of Intellectual Property (IP) for malicious gains, camouflaging of logic gates is becoming very important. In this paper, we present experimental demonstration of transistor threshold voltage-defined switch [2] based camouflaged logic gates that can hide six logic functionalities i.e. NAND, AND, NOR, OR, XOR and XNOR. The proposed gates can be used to design the IP, forcing an adversary to perform brute-force guess-and-verify of the underlying functionality-increasing the RE effort. We propose two flavors of camouflaging, one employing only a pass transistor (NMOS-switch) and the other utilizing a full pass transistor (CMOS-switch). The camouflaged gates are used to design Ring-Oscillators (RO) in ST $65 \mathrm{~nm}$ technology, one for each functionality, on which we have performed temperature, voltage, and process-variation analysis. We observe that CMOS-switch based camouflaged gate offers a higher performance $(\sim 1.5-8 \mathrm{X}$ better) than NMOS-switch based gate at an added area cost of only $5 \%$. The proposed gates show functionality till $0.65 \mathrm{~V}$. We are also able to reclaim lost performance by dynamically changing the switch gate voltage and show that robust operation can be achieved at lower voltage and under temperature fluctuation.
\end{abstract}

\section{CCS CONCEPTS}

- Security and privacy $\rightarrow$ Tamper-proof and tamper-resistant designs; • Hardware $\rightarrow$ Combinational circuits;

\section{KEYWORDS}

Reverse engineering, camouflage gate, threshold voltage-defined switch, ring-oscillator.

\section{ACM Reference format:}

Anirudh S Iyengar, Deepak Vontela, Ithihasa Reddy, Swaroop Ghosh, Syedhamidreza Motaman and Jae-won Jang. 2018. Threshold Defined Camouflaged Gates in 65nm Technology for Reverse Engineering Protection.

Permission to make digital or hard copies of all or part of this work for personal or classroom use is granted without fee provided that copies are not made or distributed for profit or commercial advantage and that copies bear this notice and the full citation on the first page. Copyrights for components of this work owned by others than ACM must be honored. Abstracting with credit is permitted. To copy otherwise, or republish, to post on servers or to redistribute to lists, requires prior specific permission and/or a fee. Request permissions from Permissions@acm.org.

ISLPED '18, July 23-25, 2018, Seattle, WA, USA

(C) 2018 Association for Computing Machinery.

ACM ISBN 978-1-4503-5704-3/18/07...\$15.00

https://doi.org/10.1145/3218603.3218641
In Proceedings of ISLPED '18: International Symposium on Low Power Electronics and Design (ISLPED '18). ACM, New York, NY, USA, 6 pages. https://doi.org/10.1145/3218603.3218641

\section{INTRODUCTION}

One of the biggest threats to hardware Intellectual Property (IP) is reverse engineering (RE). RE today has become a powerful tool for IP piracy and counterfeiting. Although techniques such as watermarking and fingerprinting [1] have been used to curb the spread of counterfeit products, they do not increase the complexity of RE itself. RE involves de-packaging the chip, milling down layerby-layer, take image of each metal layer and stitch the images together to identify the logic functionality and connectivity. The objective is to unlock the IP and clone the design.

Camouflaging is a technique of hiding the circuit functionality of a few chosen gates to make RE impossible or extremely hard [2-10]. Various flavors of camouflaging have been previously proposed (Table-I). The gate camouflaging using hollow vias [4] realizes three functions with a $\sim 5 \mathrm{X}$ area and power overhead. Aside from requiring a process change (e.g., hollow via), this technique fails to force the adversary to resort to exhaustive RE. Techniques to deceive the attacker using filler cells [5] and dummy transistors [6] are also proposed. Other obfuscation techniques $[7,8]$ suffer from either extensive signal routing and/or process change. In order to increase the RE difficulty, the threshold voltage (VT) modulation (implemented by changing channel doping concentration during manufacturing) of switches have been proposed [2,3,10]. A threshold defined switch-based multiplexer is proposed to camouflage interconnects [9]. However, it incurs routing overhead. Table-I consolidates the power delay and area overheads of the proposed methodology along with the previous discussed methods from literature. It must be noted that our proposed design is upsized and is compared to an upsized NAND-gate. We observe a $\sim 33 \mathrm{X}$ and $\sim 14 \mathrm{X}$ delay overhead between the NMOS-switch based and CMOS-switch based NAND-RO to the reference NAND-RO design. Due to the upsized nature, the power drawn by the two camouflage flavors are $22 \%$ and $17 \%$ lower than the reference NAND design.

We illustrate two generic gates (detailed in Section 2.2) that can exhibit 6 functionalities based on the $\mathrm{V}_{\mathrm{T}}$ of the switches. Unlike the existing camouflaging techniques, the VT programmable technique does not add process cost and leave no layout clues. Since proposed camouflaged gates are static logic based, they can be easily integrated with the current Electronic Design Automation (EDA) tools to provide a seamless and effective implementation.

In this paper, we present experimental demonstration of the static $\mathrm{V}_{\mathrm{T}}$ defined camouflaging technique [2,3] by analyzing each of the six 

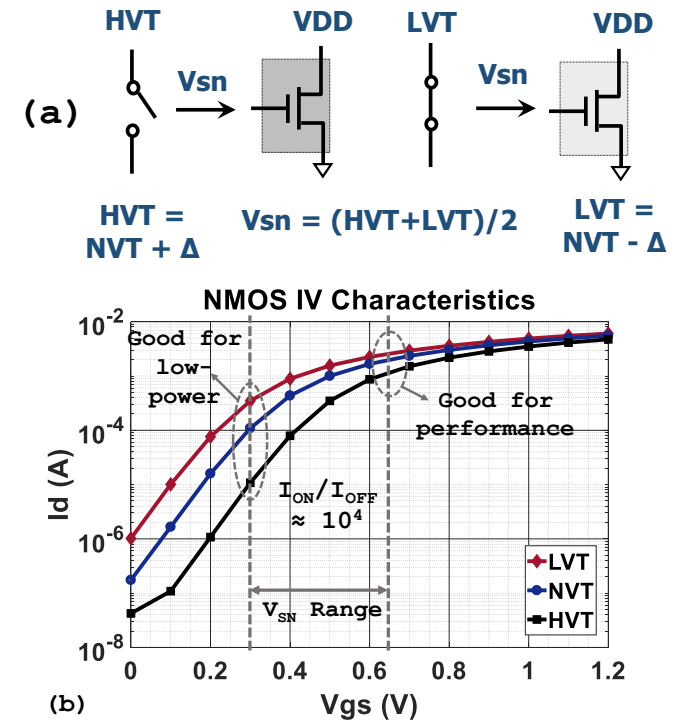

Figure 1: (a) VT programmable NMOS switch. HVT: OFF, LVT: ON. PMOS switch works similarly; (b) The I-V plot for the HVT, NVT and LVT switches biased at VSN $=($ HVT + LVT $) / 2$.

logic functionalities as Ring Oscillators (RO). Two flavors of camouflaged gates are proposed namely, NMOS-switch based, and CMOS-switch based camouflaged gates. To the best of our knowledge, this is the first experimental demonstration of $V_{T}$ defined camouflaged gates.

Adversarial assumptions: The proposed camouflaged gates are effective against common adversaries who have access to, (i) multiple copies of the chip to perform invasive RE, and use as the golden chip, (ii) RE tools e.g., access to high precision optical imaging and X-ray imaging equipment, (iii) partially correct netlist with some camouflaged gates with unknown functionality; (iv) knowledge that 6-functions can be assumed the camouflaged gates; (v) functional simulators and test pattern generators to generate patterns for a specific guess about the hidden gate function and validate their guess by applying the pattern to both golden chip and the netlist with the guessed gate function. We assume that adversary does not have access to expensive equipment to probe $\mathrm{I}-\mathrm{V}$ characteristics of the individual transistors. Since each camouflaged gate contains $8 \mathrm{~V}_{\mathrm{T}}$ defined switches (Fig. 2(a)) and an IP may contain 1-5\% camouflaged gates the number of transistors for individual characterization can be prohibitively expensive and time intensive.

Attack Model: The attacker will try all combinations of gate functionalities one-by-one and match the netlist response to validate each guess with the golden chip. With each added camouflaged gate in the design, the brute force attack time will ramp up exponentially. While the transistor threshold voltages are not directly apparent from common RE techniques like delayering and imaging the IC, there are various methods for measuring the channel doping in literature such as, spreading resistance profiling, secondary ion mass spectrometry, scanning capacitance microscopy, kelvin force probing microscopy, and electron holography [11-15]. However, these techniques have limitations in both spatial resolution and accuracy [16-19]. Even if the available techniques could provide needed resolution and accuracy, probing $\mathrm{V}_{\mathrm{T}}$ makes the RE process highly sophisticated and more resource intensive. Therefore, economics of RE with VT probing by sophisticated technique may not be justified for small ad hoc attackers.

The primary contributions of the paper are as follows:
- Demonstrate the VT defined camouflaging technique for logic obfuscation.

- Analyze the performance of the six logic functions realized as RO using both NMOS-switch and CMOS-switch based camouflaged gates.

- Perform supply voltage, temperature and process-variability analysis since $\mathrm{V}_{\mathrm{T}}$ is sensitive to these parameters.

- Demonstrate the tuning of gate biasing of $\mathrm{V}_{\mathrm{T}}$ defined switches (using $\mathrm{V}_{\mathrm{SN}}$ and/or $\mathrm{V}_{\mathrm{SP}}$ ) to not only guarantee the functionality of proposed camouflaged gates, but also reclaim lost performance at low voltages and high temperatures.

The paper is organized as follows: Section II describes the basics of $\mathrm{V}_{\mathrm{T}}$-defined switch, camouflaged gate and relevance in hardware security. Section III and IV presents the test-chip design and experimental results. Conclusions are drawn in Section V.

\section{BACKGROUND}

\subsection{Threshold defined switch [2, 3]}

The VT defined NMOS-switch (Fig. 1(a)) is realized by using an NMOS transistor which is biased with gate voltage $V_{S N}\left(V_{S N}=\right.$ $\left.0.5^{*}\left(\mathrm{~V}_{\mathrm{LVT}}+\mathrm{V}_{\mathrm{HVT}}\right)\right)$. The switch conducts when LVT is assigned on it and stops conducting when HVT is assigned. A similar process is adopted for the PMOS switch biased at VSP. Fig. 1(b) highlights the variation of current for HVT, NVT and LVT under various switch gate voltage (VSN). The value of VSN can range from $300-650 \mathrm{mV}$, while the value of $V_{S P}$ ranges from $500-850 \mathrm{mV}$ for a $\mathrm{V}_{D D}$ of $1.2 \mathrm{~V}$. This provides the flexibility to choose between a low-power (high Ion-to-IofF ratio of $\approx 10^{4}$ ) when $V_{S N}$ is low and high-performance mode when VSN is high. The switch is employed to compose multi-function gate whose functionality is selected through $\mathrm{V}_{\mathrm{T}}$ assignment.

\subsection{Multi-function camouflaged logic}

Fig. 2(a) shows the schematic of the proposed camouflaged gate that exhibits 6-functionalitites (AND, OR, NAND, NOR, XOR and XNOR) depending on the $\mathrm{V}_{\mathrm{T}}$ of switches S1-S8. The switches (S1-S8) of selected (unselected) function are programmed to LVT (HVT) whereas the input and output buffers are programmed using NVT. This design is based on NMOS switch as pass transistor in the

Table 1: Comparative Analysis of Gate Camouflaging Techniques

\begin{tabular}{|c|c|c|c|c|c|c|}
\hline 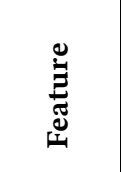 & 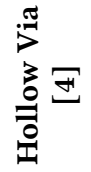 & 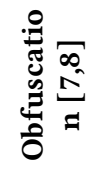 & 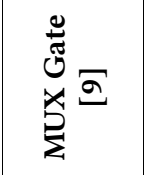 & 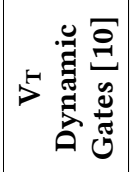 & 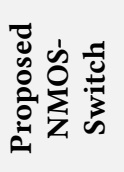 & 总离 \\
\hline$\#$ of fn. & 3 & Varies & $\begin{array}{c}2^{2^{\wedge} \mathrm{m}} \text { for } \\
2^{\mathrm{m}}: 1 \mathrm{MUX}\end{array}$ & 2 & 6 & 6 \\
\hline Area & $3.06 \mathrm{X}$ & $1.15 \mathrm{X}$ & $1.15 \mathrm{X}$ & $4.25 \mathrm{X}$ & $2.89 \mathrm{X}^{* *}$ & $3.05 X^{* *}$ \\
\hline Delay & $1.32 \mathrm{X}$ & $0.99 \mathrm{X}$ & $1.48 \mathrm{X}$ & 11.48X & $\sim 33 \mathrm{X}^{* *}$ & $\sim 14 \mathrm{X} * *$ \\
\hline Power & $3.67 \mathrm{X}$ & $1.16 \mathrm{X}$ & N/A & $3.83 \mathrm{X}$ & $0.78 \mathrm{X}^{* *}$ & $0.83 \mathrm{X}^{* *}$ \\
\hline $\begin{array}{c}\text { Static / } \\
\text { dynamic }\end{array}$ & Static & Static & Static & Dynamic & Static & Static \\
\hline $\operatorname{Sim} / \operatorname{Exp}$ & Sim & Sim & Sim & Sim & $\operatorname{Exp} * *$ & $\operatorname{Exp} * *$ \\
\hline
\end{tabular}

Note: ${ }^{* *}$ experimental comparative analysis of an upsized NAND$R O$ (as fabricated) for the reference, NMOS and CMOS-based designs.

Note: All other comparisons are performed with respect to a standard NAND gate. 
camouflaged logic. For example, a NAND logic can be realized by asserting LVT on switches S2 and S7 and HVT on all other switches. This leads to a parallel connection of PMOS transistors and a series connection of NMOS transistors. The design can be optimized to either low power or high-performance by; (i) appropriately tuning the $\mathrm{V}_{\mathrm{T}}$ of the HVT and LVT transistors; (ii) modulating the $\mathrm{V}_{\mathrm{SN}}$ and VSP voltages; and, (iii) sizing the transistors accordingly. Note that the performance and area of the proposed camouflaged gate is strongly correlated to the resistance of $\mathrm{V}_{\mathrm{T}}$ defined switches in the path. The CMOS-based camouflage logic is achieved by replacing switches S1S2 and S4-S8 with full-transmission gates (i.e., NMOS and PMOS switches in parallel). The CMOS-based switch provides full conduction of $\mathrm{V}_{\mathrm{DD}}$ and GND at the cost of higher design overhead.

\subsection{Application in Hardware Security}

Due to the overheads incurred (Table 1), the proposed camouflage gates must be used carefully. For example, swapping large gates with camouflaged gates to minimize area overhead, swapping low activity factor gates to minimize power overhead and swapping off-critical path gates to minimize delay overhead. The gates with least controllability and observability are potential candidates to be replaced with camouflaged gates to magnify the RE effort. Therefore, a trade-off exists between the overheads and security (i.e., RE effort). Note that adversary will be able to locate the camouflaged gates due to their unique appearance however, they will not know the functionality. Adversary may also be able to locate the switch gate voltage (VSN/VSP) however, they don't reveal the functionality. Probing of $\mathrm{V}_{\mathrm{SN}} / \mathrm{V}_{\mathrm{SP}}$ voltage level will not provide any clue either. VsN/VsP are DC signals and could be routed along with power rail. Unlike power rail VSN/VSP do not drive load. Therefore, the routing overhead could be kept minimal by using thin tracks.

\section{TEST-CHIP OVERVIEW}

\subsection{Design}

The proposed camouflaged gates are implemented in ST-Micro $65 \mathrm{~nm}$ technology. The die-image with the design components (annotated) is shown in Fig. 2(c). Fig. 2(b) shows the block diagram of the testchip. The design is composed of three sets of 23 stage ring-oscillators (ROs). With one set being the reference (normal gate-based RO), the second set being the only NMOS-based (pass transistor) camouflaged gates and the third set being the full CMOS-based (transmission gate) camouflaged gates. Each set is composed of the six-logic functionbased camouflaged RO. For example, the camouflaged gates are configured as NAND gates in the NAND-RO. Buffers are placed inbetween each stage of RO to provide optimal swing. Additionally, the above sets (and ROs) are power-gated to ensure only the set being currently used is selected (turned ON). The output of all the sets are MUXed to a single output pin. The $\mathrm{V}_{\mathrm{T}}$ switch voltage (V $\mathrm{V}_{\mathrm{SN}}$ and $\mathrm{V}_{\mathrm{SP}}$ ) are generated via a resistance ladder as shown in Fig. 2(b). A total of 8 voltages settings are present for both $\mathrm{V}_{S N}$ and $V_{S P}$, with $V_{S N}$ ranging from $300 \mathrm{mV}$ to $650 \mathrm{mV}$ and Vsp ranging from $500 \mathrm{mV}$ to $850 \mathrm{mV}$ (for a supply voltage of $1.2 \mathrm{~V}$ ) with a $50 \mathrm{mV}$ step. Fig. 3 shows the layout of a standard NAND, NMOS-switch based camouflaged gate and CMOSswitch based camouflaged gate used in the test-chip. The $V_{T}$ defined switches are enlarged to reduce process variation induced $V_{T}$ shift.

\subsection{Test features}

We have incorporated power gating for each set along with their corresponding ROs in the design (Fig. 2(b)). This allows us to analyze the power drawn by only by the RO that is currently active. Additionally, a scan-chain implementation (Fig. 2(b)) is employed to correctly assert the necessary control signals and the select signals to
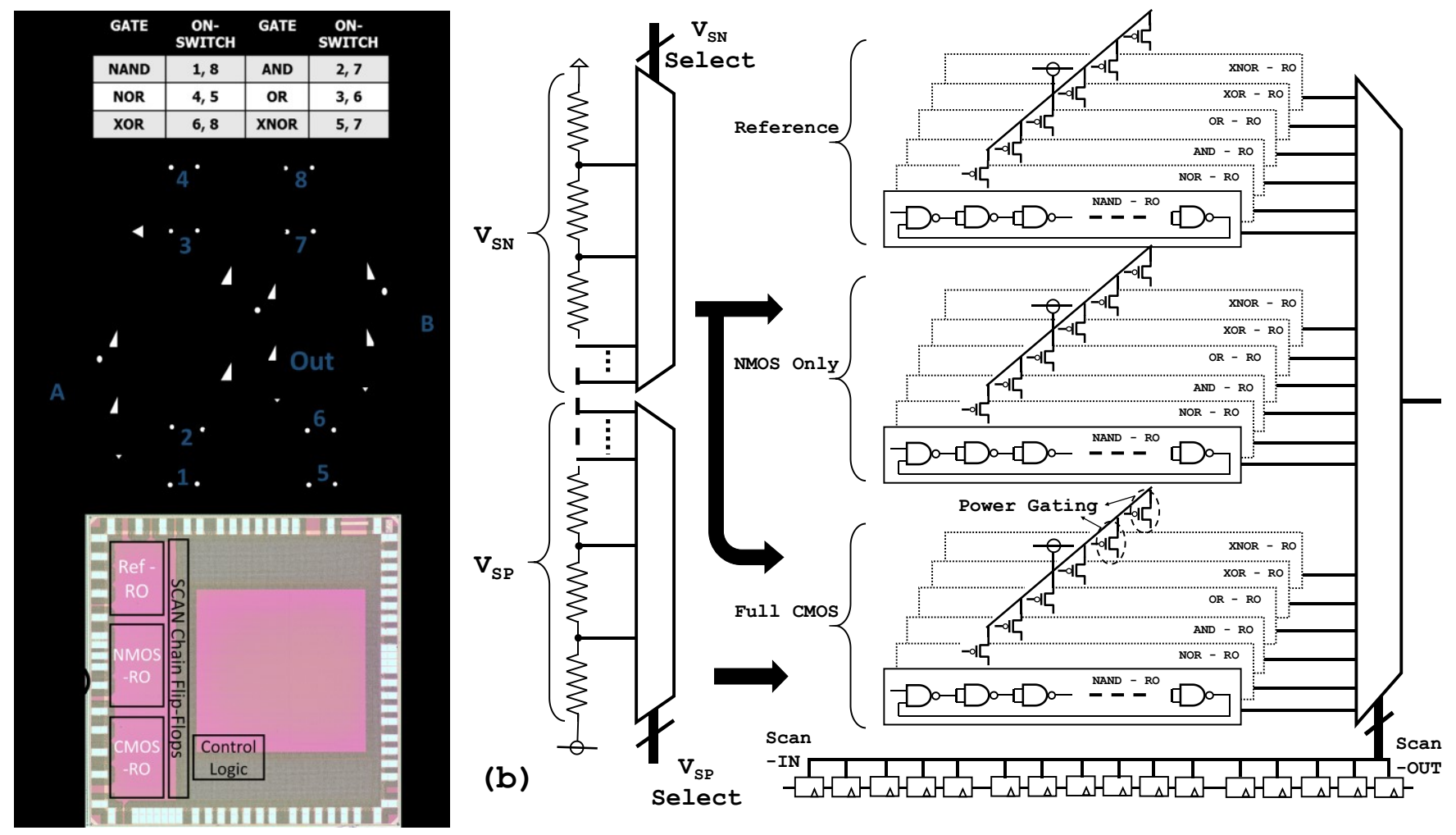

Figure 2: (a) NMOS-switch based camouflaged gate to hide 6 functionalities; (b) schematic overview of the test-chip design with a resistance ladder for generating VSN and VSP voltages; and, (c) die-image of the test-chip. 
(a)
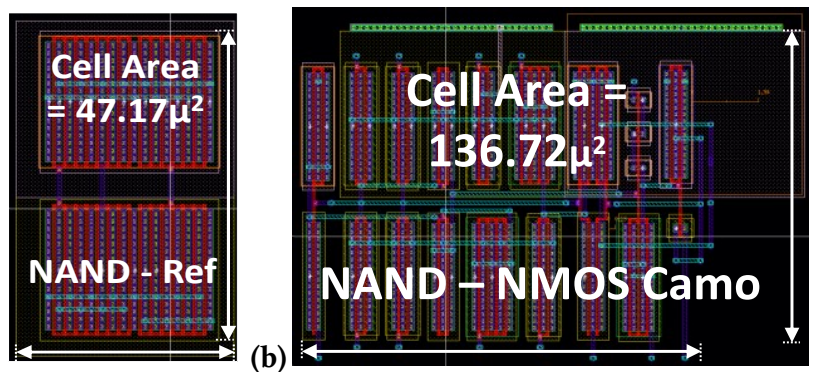

(c)

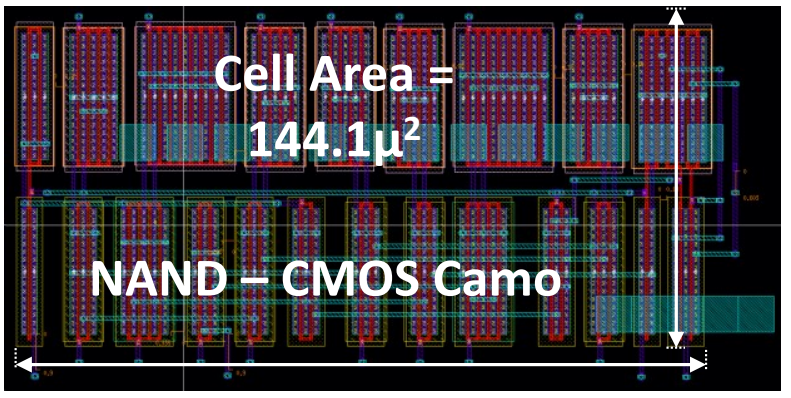

Figure 3: Layout design of: (a) reference NAND gate, (b) NMOS-switch based camouflaged gate and, (c) CMOS-switch based camouflaged gate (the gates are upsized to counter the ill-effects of process variations).

appropriately choose the required $\mathrm{RO}$ with the necessary settings (i.e. $\mathrm{V}_{S N}, \mathrm{VSP}_{\mathrm{SP}}$. We have added flexibility to dynamically select $\mathrm{V}_{\mathrm{SN}}$ and $\mathrm{V}_{\mathrm{SP}}$ (to tune camouflaged RO frequency) during-test. Thus, allowing us to control the behavior of our ROs, to achieve the best performance.

\section{EXPERIMENTAL RESULTS}

\subsection{Basic setup}

The experimental setup is composed of a logic analyzer to feed in the input stream to be scanned in; a high-sampling oscilloscope-to accurately analyze the oscillations observed; a dc-power supply and the test-chip (as shown in Fig. 4). The oscilloscope capture of NMOSswitch based NAND camouflaged gate oscillation is also depicted.

\subsection{Optimal VSN and/or VSP}

The camouflaged switches are controlled by $\mathrm{VSN}_{\mathrm{SN}}$ (NMOS) and VSP (PMOS). Fig. 5(a) shows the impact of $V_{S N}$ on the frequency of the NMOS-based camouflaged switch. We note that $500 \mathrm{mV}$ is the optimal VSN for the NMOS based switch at $1.2 \mathrm{~V}$. This is because $500 \mathrm{mV}$ provides the most overdrive for the LVT transistor without turning ON the HVT transistors. Similarly, the oscillation frequency of the CMOS-based switch for different $V_{S N}$ and $V_{S P}$ is shown in Fig. 5(b). From this plot, we observe that $450 \mathrm{mV}$ for $\mathrm{V}_{S \mathrm{~N}}$ and $600 \mathrm{mV}$ for VSP are the optimal biasing points. In this case, the driving strength of the NMOS and PMOS vary, therefore, the $V_{S N}$ and $V_{S P}$ are not necessarily between their respective HVT and LVT settings.

\subsection{Vdd scaling}

Fig. $6(a, b)$ illustrate the impact of VDD scaling on the NMOS-switch and CMOS-switch based RO. Optimal VSN and VSP are used for obtaining this data. Supply voltage scaling impacts the $V_{S N}$ and $V_{S P}$ of the $\mathrm{V}_{\text {T }}$ switches due to the shift in the node voltages of the resistance ladder. Therefore, the oscillations die out at $\sim 650 \mathrm{mV} V_{D D}$. We sweep VsN to $325 \mathrm{mV}$ at $\mathrm{VDD}_{\mathrm{DD}}=650 \mathrm{mV}$ (seen in Fig. 6(c)) and observe that the oscillation frequency increases with the increase in
$\mathrm{V}_{\mathrm{SN}}$ (bringing it closer to original optimal value). It must be noted that at $325 \mathrm{mV}$ both the HVT and LVT NMOS'es are OFF. However, the LVT is 'less' OFF than the HVT, thus resulting in the increase of frequency with the increase in $\mathrm{V}_{\mathrm{SN}}$.

Next, we perform a similar analysis for the CMOS-based RO at $\mathrm{V}_{\mathrm{DD}}=$ $650 \mathrm{mV}$. Fig. 7 illustrates the variation of oscillating frequency with respect to VSN and VSP. The best-case frequency is observed for maximum $V_{S N}$ and minimum $V_{S P}$ i.e. $V_{S N}$ of $325 \mathrm{mV}$ and $V_{S P}$ of $250 \mathrm{mV}$. This is due to the fact that at max VSN and min VSP, the LVT NMOS and PMOS are 'less' OFF than their HVT counterparts. We also note that for minimum VSN and maximum VSP, the oscillations fail. The above-mentioned tuning method can be extended to different $V_{D D}$, providing the necessary flexibility (performance to power) in selecting the bias voltage for a given $V_{D D}$.

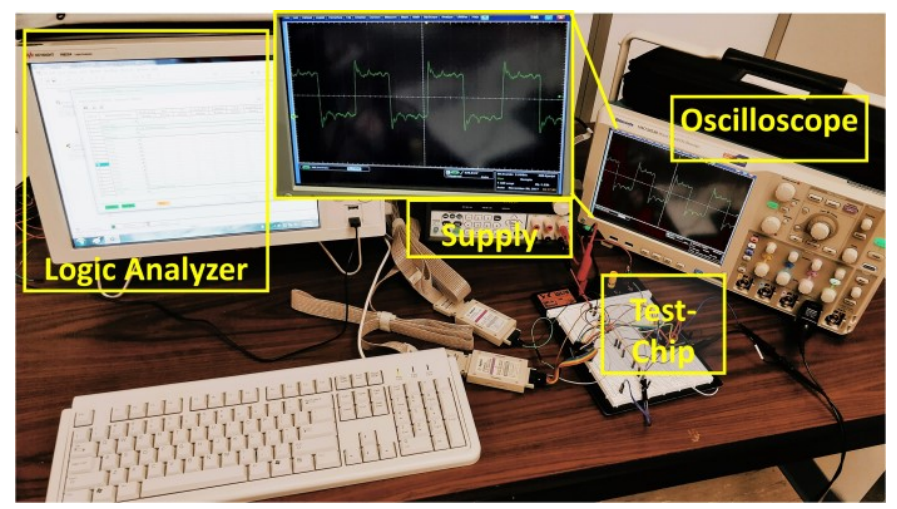

Figure 4: Test setup consisting of (i) the test chip, (ii) a logic analyzer, (iii) an oscilloscope and (iv) a power supply. The oscilloscope capture of RO oscillations of camouflaged NAND gate is also shown.

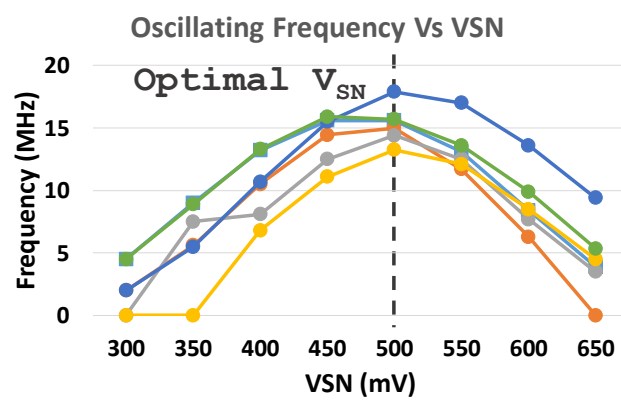

(a)

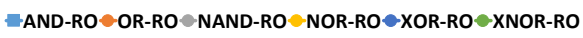

$$
\text { Variation of Freq w.r.t. Vsn and Vsp }
$$$$
\because 0-5 \square 5-10=10-15 \backsim 15-20=20-25
$$

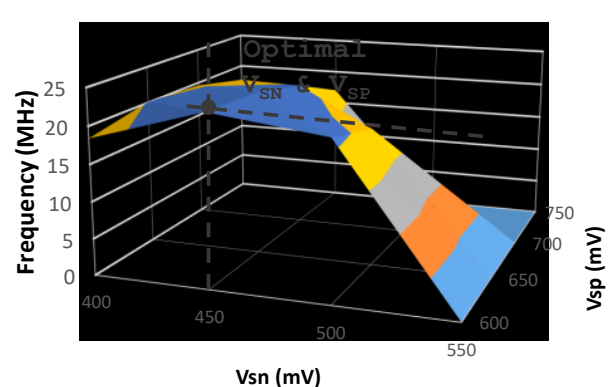

(b)

Figure 5: Frequency variation with respect to (a) VSN - for NMOS-switch (optimal VSN $=500 \mathrm{mV}$ ) and, (b) VSN + VSP for CMOS-switch (optimal VSN $=450 \mathrm{mV}$ and VSP $=600 \mathrm{mV}$ ). 


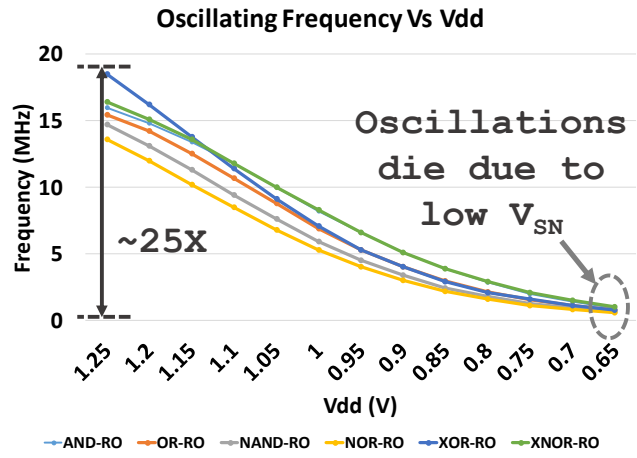

(a)

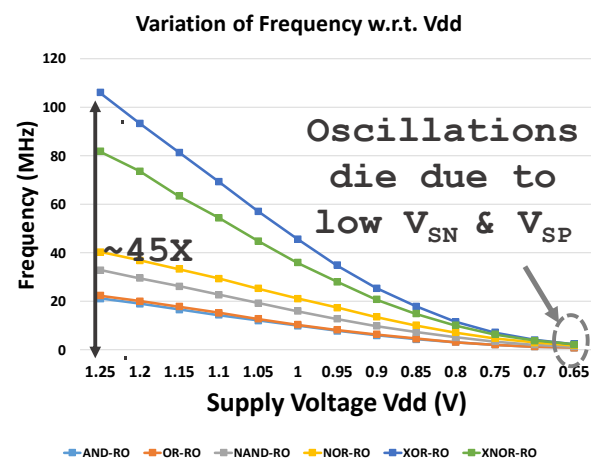

(b)

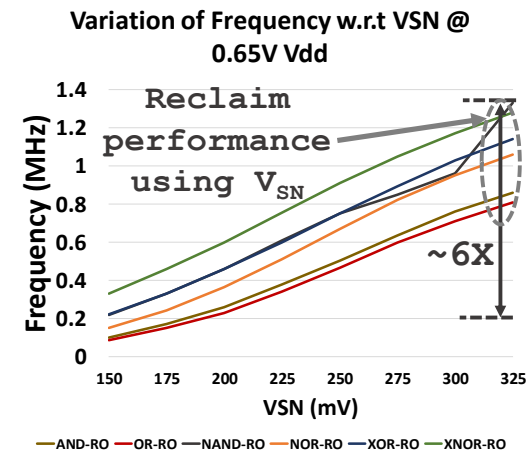

(c)

Figure 6: Frequency variation with respect to (a) VDD for NMOS-switch @ VSN=500mV, (b) VDD for CMOS-switch @ VSN= $450 \mathrm{mV}$ and VSP $=600 \mathrm{mV}$, and, (c) VSN at $650 \mathrm{mV}$ VDD. It must be noted that resistance ladder voltage changes with VDD, thus leading to a smaller VSN step size.

Variation of Frequency w.r.t. Vsn and Vsp @650mV Vdd

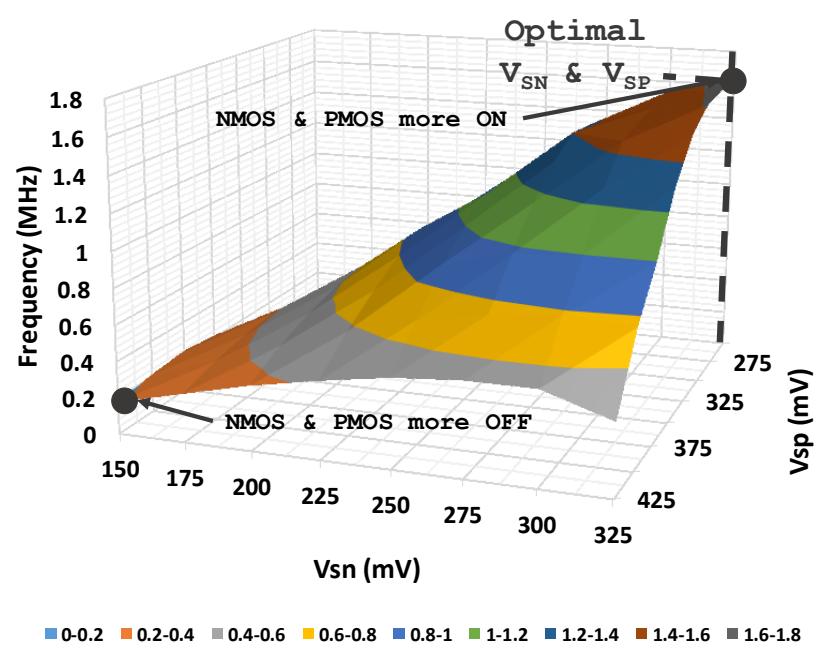

Figure 7: Optimal VSN and VSP for CMOS-switch at $650 \mathrm{mV}$ VDD (optimal VSN $=325 \mathrm{mV}$ and VSP $=275 \mathrm{mV}$ ).

\subsection{Process variations}

We have analyzed the frequency response of the NMOS-switch and CMOS-switch based camouflaged gates for 10 test-chips (Fig. 8(a)). We observe $\sim 5 \%$ variation in the frequency distribution for each function. A $5 \mathrm{X}$ to $7 \mathrm{X}$ improvement in the speed is observed between the CMOS-switch to the NMOS-switch based camouflaged gates. The designs exhibit less sensitivity to process variation due to the enlarged switch sizes. The experimental results indicate that the proposed gates are robust with respect to process variations.

\subsection{Temperature variation}

Temperature has a direct impact on the performance of the camouflage gates since VT is a function of temperature. Fig. 8(b) illustrates the impact of temperature on the oscillation frequency of the NMOS switch-based RO. With the increase in temperature, the transistor's $\mathrm{V}_{\mathrm{T}}$ reduces, which correspondingly shifts the HVT and LVT values. If left unchecked, the OFF switches with HVT will turn ON. This in turn will start contending with internal signals and corrupts the functionality. Therefore, VSN and VSP need to be adjusted appropriately to restore the optimal functionality. This is illustrated in Fig. 9, where the device is heated to a temperature of $\sim 65^{\circ} \mathrm{C}$ and the
$V_{\text {SN }}$ bias is swept from $300 \mathrm{mV}$ to $650 \mathrm{mV}$ to find the optimal bias point for a NMOS-based RO. It must be noted that the $\mathrm{V}_{\mathrm{T}}$ of the device reduces by $\sim 2 \mathrm{mV}$ for each degree rise in temperature, which would therefore shift the VT of the device by $\sim 80 \mathrm{mV}$ at $65^{\circ} \mathrm{C}$. From the experiment, we observe that the optimal $\mathrm{V}_{\mathrm{SN}}$ bias point has shifted from $500 \mathrm{mV}$ at $25^{\circ} \mathrm{C}$ to $450 \mathrm{mV}$ at $65^{\circ} \mathrm{C}$. This result indicates that bias voltage can be optimized to counter the effects of temperature. All modern processors include temperature sensors. The temperature and supply voltage combinations can be used to select appropriate VSN and VSP settings for the robust operation of the camouflaged gates.

\section{DISCUSSION}

\subsection{Security Analysis}

Although the proposed camouflaging is obtained free of cost due to multi- $\mathrm{V}_{\mathrm{T}}$ feature offered in advanced nodes, they are sensitive to temperature which is a vulnerability. The adversary can use temperature to gain insights about the camouflaged gate functionality since each individual gate flavor provide different delay signature with temperature. However, modulation of $\mathrm{V}_{\mathrm{SN}} / \mathrm{V}_{\mathrm{SP}}$ of switches under temperature variation can eliminate the side channel signature.

\subsection{Integration with EDA tools}

The proposed technique adds minimal changes to the EDA flow. First, the standard cell library is modified to include the NMOS-switch based and CMOS-switch based camouflaged gates. This is achieved by creating a liberty file of the above two flavors of camouflage gates. The values used in the liberty file are populated by characterizing the gates in terms of area, delay and power. Then, tools such as, Synopsys dc shell [20] can be employed to perform in-depth design, analysis and synthesis of any combinational logic design that utilized the two flavors of camouflage gates. Finally, appropriate layout files (LEF/GDS) are developed and made available to the physical designers for implementation.

\section{CONCLUSION}

We demonstrate VT switches to hide six logic functionalities and proposed two camouflaged gates i.e. NMOS-switch and CMOSswitch based design experimentally. The CMOS-switch offered higher performance with only a minimal increase in area. Biasing knobs i.e. $V_{S N}$ and $V_{S P}$ are used to study the impact on performance with supply voltage scaling and temperature variation and can be tuned dynamically to combat ill effects of temperature, voltage and 


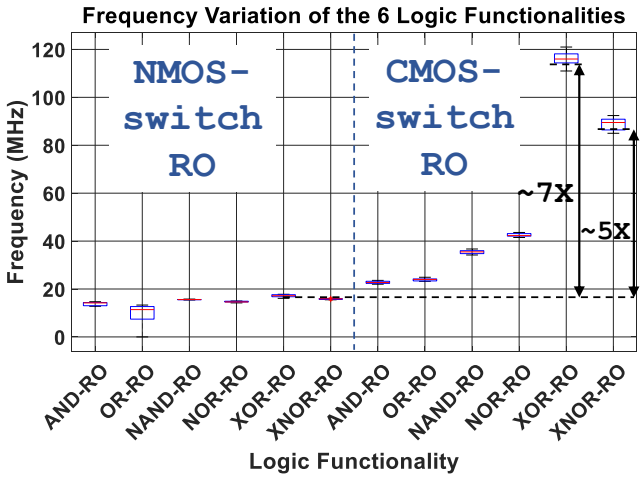

(a)

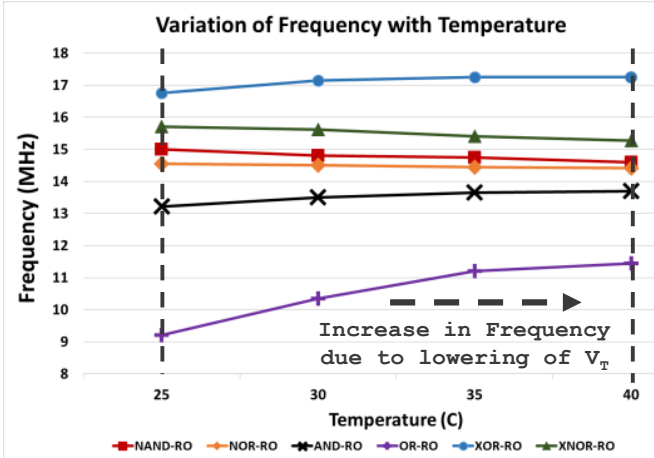

(b)

Figure 8: (a) Frequency distribution of 10 die for both the NMOS-switch and CMOS-switch based RO; (b) variation of oscillating frequency under change in temperature for an NMOS-switch based RO.

\section{NAND-RO w.r.t VSN @ 25C and 65C}

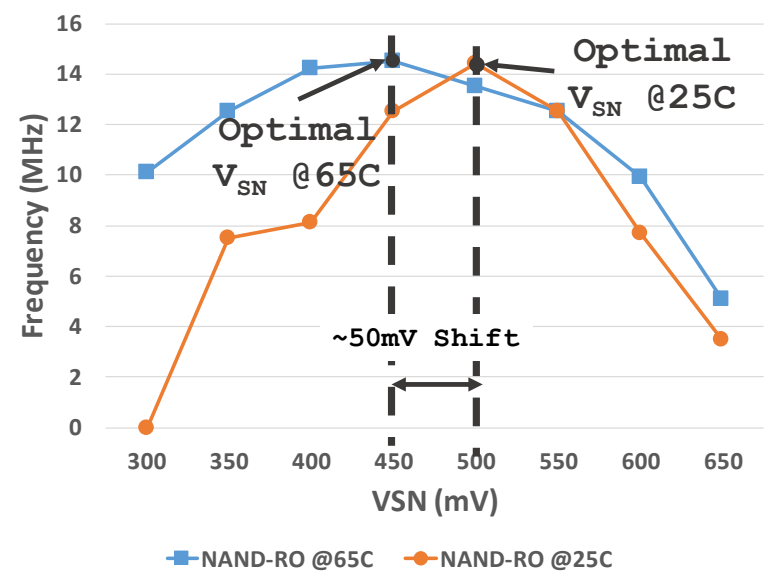

Figure 9: Optimal VSN bias shift under the effect of temperature $\left(65^{\circ} \mathrm{C}\right)$.

process variations. The proposed design does not leave any logic or physical level clues, and when used judiciously, improves the overall RE effort.

\section{ACKNOWLEDGMENT}

This work was supported by NSF (\#CNS-1722557, \#CCF-1718474 and \#DGE-1723687), and DARPA Young Faculty Award (\#D15AP00089).

\section{REFERENCES}

[1] Charbon, Edoardo. "Hierarchical watermarking in IC design." In Custom
Integrated Circuits Conference, 1998. Proceedings of the IEEE 1998, pp. 295-298. IEEE, 1998.

[2] Nirmala, Ithihasa Reddy, Deepak Vontela, Swaroop Ghosh, and Anirudh Iyengar. "A novel threshold voltage defined switch for circuit camouflaging." In Test Symposium (ETS), 2016 21th IEEE European, pp. 1-2. IEEE, 2016.

[3] De, Asmit, and Swaroop Ghosh. "Preventing Reverse Engineering using threshold voltage defined multi-input camouflaged gates." In Technologies for Homeland Security (HST), 2017 IEEE International Symposium on, pp. 1-6. IEEE, 2017.

[4] Rajendran, Jeyavijayan, Michael Sam, Ozgur Sinanoglu, and Ramesh Karri. "Security analysis of integrated circuit camouflaging." In Proceedings of the 2013 ACM SIGSAC conference on Computer \& communications security, pp. 709-720. ACM, 2013..

[5] Chow, Lap Wai, James P. Baukus, Bryan J. Wang, and Ronald P. Cocchi. "Camouflaging a standard cell based integrated circuit." U.S. Patent 8,151,235, issued April 3, 2012.

[6] Baukus, James P., Lap-Wai Chow, and William M. Clark Jr. "Method and apparatus using silicide layer for protecting integrated circuits from reverse engineering." U.S. Patent 6,117,762, issued September 12, 2000.

[7] Chakraborty, Rajat Subhra, and Swarup Bhunia. "Security against hardware Trojan attacks using key-based design obfuscation." Journal of Electronic Testing 27, no. 6 (2011): 767-785.

[8] Chakraborty, Rajat Subhra, and Swarup Bhunia. "Security against hardware Trojan through a novel application of design obfuscation." In Computer-Aided Design-Digest of Technical Papers, 2009. ICCAD 2009. IEEE/ACM International Conference on, pp. 113-116. IEEE, 2009.

[9] Jang, Jae-Won, and Swaroop Ghosh. "A Novel Interconnect Camouflaging Technique using Transistor Threshold Voltage." arXiv preprint arXiv:1705.02707 (2017).

[10] Collantes, Maria I. Mera, Mohamed El Massad, and Siddharth Garg. "Threshold-dependent camouflaged cells to secure circuits against reverse engineering attacks." In VLSI (ISVLSI), 2016 IEEE Computer Society Annual Symposium on, pp. 443-448. IEEE, 2016.

[11] Vandervorst, Wilfried, Trudo Clarysse, and Pierre Eyben. "Spreading resistance roadmap towards and beyond the $70 \mathrm{~nm}$ technology node." Journal of Vacuum Science \& Technology B: Microelectronics and Nanometer Structures Processing, Measurement, and Phenomena 20, no. 1 (2002): 451-458

[12] Duhayon, Natasja, Pierre Eyben, Marc Fouchier, Trudo Clarysse, Wilfried Vandervorst, David Álvarez, S. Schoemann et al. "Assessing the performance of two-dimensional dopant profiling techniques." Journal of Vacuum Science \& Technology B: Microelectronics and Nanometer Structures Processing, Measurement, and Phenomena 22, no. 1 (2004): 385-393.

[13] Williams, C. C. "Two-dimensional dopant profiling by scanning capacitance microscopy." Annual review of materials science29, no. 1 (1999): 471-504.

[14] Sommerhalter, Ch, Th W. Matthes, Th Glatzel, A. Jäger-Waldau, and M. Ch Lux-Steiner. "High-sensitivity quantitative Kelvin probe microscopy by noncontact ultra-high-vacuum atomic force microscopy." Applied Physics Letters 75, no. 2 (1999): 286-288

[15] Völkl, Edgar, Lawrence F. Allard, and David C. Joy, eds. Introduction to electron holography. Springer Science \& Business Media, 2013.

[16] Huang, Y., C. C. Williams, and H. Smith. "Direct comparison of crosssectional scanning capacitance microscope dopant profile and vertical secondary ion-mass spectroscopy profile." Journal of Vacuum Science \& Technology B: Microelectronics and Nanometer Structures Processing, Measurement, and Phenomena 14, no. 1 (1996): 433-436.

[17] Vartanian, V., M. Sadaka, S. Zollner, AV-Y. Thean, T. White, B-Y. Nguyen, M. Zavala et al. "Metrology Challenges for $45 \mathrm{~nm}$ Strained-Si Devices." In AIP Conference Proceedings, vol. 788, no. 1, pp. 214-221. AIP, 2005.

[18] M. V. Stangoni, "Scanning Probe Techniques for Dopant Profile Characterization," in $\mathrm{PhD}$ Thesis, 2005.

[19] De Wolf, Peter, Robert Stephenson, Thomas Trenkler, Trudo Clarysse, Thomas Hantschel, and Wilfried Vandervorst. "Status and review of two-dimensional carrier and dopant profiling using scanning probe microscopy." Journal of Vacuum Science \& Technology B: Microelectronics and Nanometer Structures Processing, Measurement, and Phenomena 18, no. 1 (2000): 361-368.

[20] Synopsys design compiler: https://www.synopsys.com/ support/training/rtl-synthesis/design-compiler-rtl-synthesis.html 